\title{
Entrevista com Susanna Witt: História da tradução soviética foi assunto sensível e, por isso, negligenciado
}

Marina Darmaros

Susanna Witt tem formação em Estudos Eslavos e defendeu seu doutorado, em 2001, sobre a criação de Doutor Jivago por Pasternak, na Universidade de Estocolmo, onde leciona atualmente. De lá para cá, enveredou nos caminhos dos Estudos da Tradução, área de pesquisa sobre a qual se debruça atualmente, dedicando-se principalmente à era soviética.

1 - Conte-nos um pouco sobre sua trajetória e como começou nos Estudos da Tradução russos. Foi um caminho dificil nos Estudos da Tradução voltados especificamente para a realidade russo-soviética?

Tenho formação em Estudos Eslavos, com especialização em literatura russa. Defendi meu doutorado, Criando a Criação: Leituras de Doutor Jivago, de Pasternak [tradução nossa] na Universidade de Estocolmo em 2001 e comecei a publicar na área de modernismo literário russo. Borís Pasternak também era um tradutor de renome e, mais tarde, escrevi um artigo sobre sua versão de Hamlet, de Shakespeare. Como resultado, interessei-me por uma significação mais ampla da tradução literária como fenômeno cultural na Rússia e, particularmente, na União Soviética, mas fiquei surpresa ao descobrir que o assunto era, em grande medida, negligenciado nos Estudos Eslavos (ocidentais) e praticamente inexistente das

1 Creating Creation: Readings of Pasternak's Doktor Zhivago. 
principais publicações de Estudos da Tradução modernos. Isto ocorreu por volta de meados da década de 2000. Na Rússia em si tem havido, claro, uma longa e forte tradição de estudos e teorização da tradução, mas ela é monolinguisticamente russófona e, durante os tempos soviéticos, frequentemente teve uma abordagem normativa e severamente ideologizada. Por motivos ideológicos, a história da tradução daquele período era um assunto sensível e, por isso, negligenciado. A pesquisa da tradução do final da União Soviética e do período pós-soviético se baseou em grande parte na linguística e teve caráter prescritivo. Essa cegueira foi a razão pela qual decidi me dedicar à questão em um projeto, "Totalitarismo e Tradução: Controle e Conflito nas Práticas de Tradução Soviéticas, 1932-1953”2 [tradução nossa], voltado a estabelecer a tradução literária como objeto de pesquisa dentro dos estudos da cultura do período Stálin. Durante os anos de 2009 a 2013, trabalhei extensivamente em arquivos russos, o que foi uma nova experiência para mim - e realmente difícil, com certeza. De alguma maneira, eu senti que as coisas não haviam mudado muito desde os tempos soviéticos (durante os quais passei um ano na Universidade de Moscou), apesar da rápida e evidente modernização da cena urbana e da vida cotidiana.

2 - Em seu artigo "A Arte da Acomodação: A Primeira Conferência de Tradução da Pan-

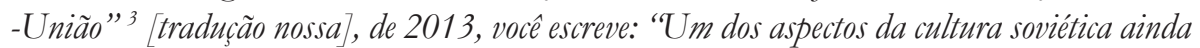
muito negligenciado, porém, é o da tradução literária". Você acredita que esses estudos ainda sejam deixados de lado ou eles estão crescendo?

Eu diria que, agora, o cenário mudou radicalmente (especialmente tendo em mente que o artigo citado por você foi escrito alguns anos antes de sua publicação, em 2013). Dentro da ampliação geral das perspectivas geográficas que os estudos da tradução têm visto nas últimas décadas (pense no grande volume de pesquisas sobre tradução relativos a Índia, China, Japão, África e Américas) e que vem desafiando as prioridades tradicionalmente eurocêntricas da disciplina, a Rússia e o Leste Europeu finalmente ganharam visibilidade. Isto ocorreu devido à colaboração internacional, conferências e iniciativas de publicação que reuniram pesquisadores de diversas disciplinas e fronteiras. Em 2008, participei da maior conferência da área, organizada por Emily Lygo na Universidade de Exeter, Grã Bretanha ("Os

2 Totalitarianism and Translation: Control and Conflict in Soviet Translation Practices, 1932-1953.

3 The art of accommodation: The First All-Union Conference of Translators. 
Post Horses de Púchkin: Tradução Literária e Cultura Russa"5 [tradução nossa]). A conferência reuniu acadêmicos da Rússia, Europa e América, muitos dos quais seriam úteis para promover a pesquisa na área, sobretudo Brian James Baer, que editou e publicou em 2011 o tomo Contextos, Entrelinhas e Pretextos: A Tradução Literária no Leste Europen e na Rússia $a^{6}$ [tradução nossa]. Também colaborei com um capítulo para o livro, apresentando os primeiros resultados de meu projeto. Já a conferência de Exeter resultou no tomo de 2013 A Arte da Acomodação: Tradução Literária na Rússia [tradução nossa] (editada por Leon Burnett e Emily Lygo). Em 2014, organizei uma conferência internacional ("Tradução em Contextos Russos: Pontos de Partida Transculturais, transliterais e Transdisciplinares") ${ }^{8}$, na Universidade de Uppsala, na Suécia, junto a minha colega Julie Hansen. Seus 60 participantes provenientes de 15 países subsequentemente compuseram o núcleo de uma rede de pesquisadores em expansão na área, que agora inclui mais de 100 pesquisadores. Um volume baseado em colaborações selecionadas da conferência de Uppsala acaba de sair (editado por Brian James Baer e Susanna Witt, Traducão em Contextos Russos: Cultura, Política, Identidade [tradução nossa]). Também gostaria de citar Andrea Ceccherelli, Lorenzo Costantino, Cristiano Diddi (eds.) Translation Theories in the Slavic Countries, Salerno 2015. Esta é uma edição especial da publicação Europa Orientalis, vol. XXXIII (2014), baseada em uma conferência realizada quase simultaneamente com a nossa de Uppsala, em 2014.

Como sinal de um marco revolucionário transdisciplinar, edições especiais dedicadas à Rússia estão saindo em publicações de tradução, como o Translation and Interpreting Studies (11:1, 2016) e o Translation Studies (11:2, 2018), enquanto, por exemplo, o Slavic and East European Journal deu, recentemente, destaque a um "fórum" sobre tradução na Rússia $(60: 1,2016)$. Também houve um crescimento no interesse quanto à pesquisa russa sobre tradução, como testemunhou a conferência "Rumo a Leste: Descobrindo Tradições Novas e Alternativas nos Estudos

\footnotetext{
4 Termo utilizado para designar um cavalo mantido na estalagem para mensageiros e carruagens de correio ou para aluguel a viajantes (Dicionário Collins. Acesso em 07/02/2018 https://www.collinsdictionary.com/pt/dictionary/english/post-horse). (N. do T.)

5 Pushkin's Post Horses: Literary Translation in Russian Culture.

6 Contexts, Subtexts and Pretexts: Literary Translation in Eastern Europe and Russia.

7 The Art of Accommodation: Literary Translation in Russia (eds. Leon Burnett and Emily Lygo)

8 Translation in Russian Contexts: Transcultural, Transliteral and Transdisciplinary Points of Departure.

9 Brian James Baer, and Susanna Witt (eds.), Translation in Russian Contexts: Culture, Politics, Identity, London: Routledge, 2018
} 
da Tradução"10 [tradução nossa], realizado na Universidade de Viena em 2014 (e o tomo de 2016 de mesmo título, editado por Laris Schippel e Cornelia Zwischenberger). É também digno de nota aqui o tomo editado por Birgit Mensel e Irina Alekseeva Russische Übersetzungswissenschaft an der Schwelle zum 21. Jabrbundert, que saiu em 2013. Assim, o volume de pesquisas sobre tradução e os estudos da tradução no contexto russo está aumentando continuamente (inclusive com um número crescente de teses de doutorado), apesar de haver ainda obstáculos, como os sistemas relativamente fechados de circulação: as pesquisas acadêmicas em língua francesa, alemã e inglesa ainda tendem a ignorar umas as outras, enquanto estudos baseados na Rússia frequentemente operam sem referências ao conhecimento internacional.

3 - Quais são os principais pesquisadores desta área na atualidade, na sua opinião? E quais textos você considera canônicos e recomenda a alguém que tenha já alguma base em Estudos da Tradução, mas queira enveredarpelos Estudos da Tradução Russos? Que conselhos você daria a alguém iniciando seu caminho nos Estudos da Traducão Russos?

Já citei alguns nomes anteriormente. Quando se fala em tradução no contexto russo, uma das figuras centrais é, sem dúvidas, o supracitado Brian James Baer, que publicou sua monografia Tradução e a Construção da Literatura Russa Moderna ${ }^{11}$ [tradução nossa] em 2016. Em um contexto mais amplo da história da tradução na Rússia, gostaria de citar o livro de Serguêi Tiulênev Tradução e Ocidentalizaçãa da Rússia do Século Dezoito: Uma Abordagem Sócio-Sistêmica ${ }^{12}$ (2012) [tradução nossa]. Quanto à pesquisa de arquivo (possibilitada com a quebra do sistema soviético), é preciso mencionar a monografia de Andrêi Azóv, de 2013, Literalistas derrotados: Da história da tradução literária na URSS nos anos de 1920-1960 ${ }^{13}$ [tradução nossa]. Minha própria pesquisa, parcialmente baseada no mesmo material-fonte, tem muitos pontos em comum com a dele, apesar de minha abordagem ser mais ampla e incluir análise da tradução distintiva. Há uma pesquisa interessante sendo conduzida também sobre periódicos soviéticos que apresentavam a literatura estrangeira em tradução para o russo, as publicações Internatsionálnaia literatura ${ }^{14}$ (1933-1943)

10 Going East: Discovering New and Alternative Traditions in Translation Studies.

11 Translation and the Making of Modern Russian Literature.

12 Translation and the Westernization of Eighteenth Century Russia: A Social-Systemic Approach.

13 Poverjennie bukvalisti: Iz istori khudojestvennogo perevoda v SSSR v 1920-1960-e godi.

14 Em tradução livre, "Literatura internacional". 
e Inostránnaia literatura ${ }^{15}$ (1955-). A última é objeto de um projeto de pesquisa na Escola Superior de Economia de Moscou conduzido por colegas do meu círculo, Elena Zemskova e Elena Ostrovskaia, e tem por foco os aspectos institucionais e pessoais das atividades dos periódicos. Este periódico também foi estudado por Nailya Safiullina e Rachel Polonsky, que se concentraram, entre outros, na recepção desses textos na Rússia de Stálin. Uma contribuição valiosa aqui também é a monografia de Samantha Sherry, Discursos de Regulamentação e Resistência: Censurando Traduçoes nas Eras Stálin e Khruschov da União Soviética ${ }^{16}$ [tradução nossa], um estudo comparativo de dois periódicos. Quanto a "textos canônicos", os estudos pioneiros de Maurice Friedberg (apesar de não serem baseados em arquivos) devem ser citados aqui: Uma década de Euforia. Literatura Ocidental na Rússia Pós-Stálin, 1954-64 ${ }^{17}$ (1997) [tradução nossa]. A teoria soviética da tradução é apresentada de maneira seletiva no livro também pioneiro de Lauren Leighton de 1991 Dois Mundos, Uma Arte: Tradução Literária na Rússia e na América ${ }^{18}$ [tradução nossa]. Alguns clássicos da tradução russo-soviética podem ser acessados em outras línguas: o clássico de Kornêi Tchukóvski Alta Arte ${ }^{19}$ [tradução nossa], que foi traduzido por Lauren Leighton como The Art of Translation: Kornei Chukouskii's "The High Art" (1984); Efim Etkind, um dos mais astutos pesquisadores da tradução russa, é representado em francês pelo volume Un art en crise: essai de poétique de la traduction poétique (1982, traduzido por Wladimir Troubetzkoy) ${ }^{20}$. Brian James Baer está traduzindo agora o seminal Fundamentos para uma teoria geral da tradução ${ }^{21}$ [tradução nossa] (primeira edição de 1953), de Andrêi Fiôdorov, e publicou anteriormente Escritores Russos Sobre Tradução: Uma Antologia ${ }^{22}$ [tradução nossa] (2013, juntamente com Natália Olchanskaia). No momento, eu mesma, junto com Baer, estou preparando uma "História soviética da tradução em documentos", tornando acessíveis textos do início dos anos 1920 e posteriores.

15 Em tradução livre, "Literatura estrangeira".

16 Discourses of Regulation and Resistance: Censoring Translation in the Stalin and Khrushchev Era Soviet Union.

17 A Decade of Euphoria. Western Literature in Post-Stalin Russia, 1954-64 (1977) and Literary Translation in Russia. A Cultural History.

18 Two Worlds, One Art: Literary Translation in Russia and America.

19 Intitulado originalmente em russo como Vissókoe iskússtvo.

20 Intitulado originalmente em russo como Osnovi obschei teorii perevoda ("Fundamentos de uma teoria geral da tradução").

21 Foundations for a general theory of translation.

22 Russian Writers on Translation: An Anthology. 
Meu conselho a quem "estiver trilhando seu caminho nos Estudos da Tradução russos" seria, talvez, manter-se atento ao fato de que a tradução na Rússia sempre foi "mais que tradução" (para parafrasear o dito popular na Rússia de que "um poeta é mais que um poeta"): já que a tradução é a manifestação física de um encontro com "o estrangeiro", ela sempre foi uma atividade sensível em uma sociedade que foi frequentemente informada por suspeita e se resguardou ao longo da história com regulamentações de viagem e coisas similares. Um local de negociação, contestação e resistência, a tradução russa sempre tem que ser contextualizada para poder ser conceituada. Isto, claro, diz respeito à tradução em geral, mas talvez seja ainda mais importante aqui.

4 - Em muitas de suas obras, você estuda casos que envolvem linguagens em que não é nativa, ao que me parece (como nas traduções de Djambul ou Pasternak de dialetos lequíticos etc.). Como você escolhe os casos que pesquisará, presumindo que a barreira da lingua pode ser maior neste sentido?

É verdade. Há limitações óbvias para o número de línguas que um pesquisador pode conhecer razoavelmente. Minha língua nativa é o sueco, sou fluente em russo e inglês e tenho habilidade de leitura em francês, polonês e ucraniano. Mas eu diria que tudo depende do tipo de pesquisa que você queira fazer. Os casos que você cita são muito diversos, apesar de unidos pelo fato de que são ambos mediados, e não traduções diretas. Ambos são produzidos com o auxílio de uma língua russa interlinear, o chamado podstrochnik (que significa, literalmente, "sob a linha" $)^{23}$, uma prática soviética muito comum à qual dediquei diversos estudos. Assim, os próprios tradutores soviéticos nem sempre conheciam (na realidade, mais frequentemente eles não conheciam) as línguas do original, especialmente quando isso se referia a traduções das muitas línguas da própria União Soviética. Quanto a Djambul, muitos dos textos originais em cazaque estão faltando ou são contestáveis, e meu principal foco foi a função e o significado dessas traduções em seu contexto. Eu também consegui detectar o manejo institucional deles e esboçar o provável processo de produção, que parecia ser uma empreitada coletiva resultando em traduções e também nos textos originais em cazaque. No caso da tradução de Pasternak do poeta lequítico Óndra Łysohorsky, encontrei os textos originais no arquivo de Pasternak, assim como o próprio russo interlinear de Łysohorsky.

23 Em russo, o vocábulo tem sentido de tradução literal, palavra a palavra. (N. do T.) 
Pude ler o original graças a meus conhecimentos de polonês, já que o lequítico, um dialeto que o próprio Lysohorsky promoveu a língua, é algo entre polonês, tcheco e eslovaco. Pude estimar a proximidade que o interlinear tinha do original e analisar a tradução de Pasternak a partir disso. Conduzi análises detalhadas da tradução apenas de idiomas que conheço, como a versão de Gueórgui Chengueli de Don Juan, de Byron (WITT, Susanna, "Translation and Intertextuality in the Soviet-Russian Context: The Case of Georgii Shengeli’s Don Juan.” Cluster on Russian translation, ed. by Brian James Baer. Slavic and East European Journal, 60:1, 2016).

5 - Vocêpoderia pontuar algumas das características peculiares aos Estudos da Traducão Russos que o diferem dos estudos em outras linguagens e realidades? "Literalismo", "tradução livre", "tradução realista", "naturalismo", "tradução realista" são, sob meu ponto de vista, características desconbecidas por muitos pesquisadores que não se concentram na área russa desses estudos, você concorda? Você poderia pontuar também alguns dos casos mais interessantes em Estudos da Tradução russos em sua opinião? Em suas pesquisas, você cita o caso da pseudotradução de Maimbet, on a breve discussão iniciada por Gideon Toury sobre o caso Djambul como um exemplo de "planejamento cultural"... Você poderia falar um pouco sobre isto?

Você tem razão, é claro que essas noções não são exclusivamente russas (exceto, talvez, pela "tradução realista", que, ao que eu saiba, não foi conceituada em nenhum outro lugar). Mas uma característica distintiva do contexto soviético é a idealização da norma, ligando determinadas abordagens da tradução a posições ideologicamente repreensíveis e definindo os limites do discurso da tradução. É preciso lembrar que, em certos períodos, essas questões não eram puramente acadêmicas, e podiam mandar as pessoas aos campos de trabalhos forçados.

Gideon Toury (2005) foi o primeiro a trazer Djambul, o bardo cazaque do estalinismo, à pesquisa acadêmica ocidental da tradução. Mas sua única fonte para teorizar o que ele chamou de pseudotradução neste caso foram as memórias do compositor Dmítri Chostakóvitch, um texto que é um pouco controverso em si mesmo. Não oficialmente, no contexto soviético, o caso Djambul também foi um tratamento mais completo como indicador das práticas soviéticas de tradução e atitudes quanto à tradução em um contexto imperial. No processo, descobri alguns casos similares em que poetas das "nacionalidades" 24 publicados principalmente em traduções russas eram promovidos a representantes de suas literaturas nativas

24 As diversas etnias encontradas na Rússia são chamadas, até hoje, de "nacionalidades" (natsionálnasti) dentro do país. (N. do T.) 
para serem incluídos no cânone soviético. É a isto que eu, usando o conceito de Toury e até de Even Zohar de "planejamento cultural", refiro-me como "planejamento cultural de baixo", já que os próprios tradutores (russos) eram frequentemente instrumentais em publicar esses textos. O caso de Maimbet é extremo porque não apenas não havia textos originais, mas tampouco havia um poeta original. Esta figura cazaque foi lançada em 1935 por um jornalista local russo em Alma-Ata. As traduções dele de Maimbet apareceram até no jornal central Pravda, acompanhadas de uma foto do bardo, um homem típico da Ásia Central com seu tradicional instrumento de cordas. Naquele tempo, havia uma grande demanda (criada de cima) por poetas "das nacionalidades" para representar suas repúblicas em festivais culturais nas maiores cidades russas. Assim, o jornalista foi abordado pelas autoridades, que pediam que ele trouxesse seu poeta a Moscou para o festival cazaque, em 1936. Sua solução para o problema foi anunciar que Maimbet, membro de uma família nômade, havia cruzado a fronteira para a China e ficado por lá. O status imaginário de Maimbet foi confirmado em uma carta de denúncia dos colegas do jornalista na união dos escritores local, que encontrei nos arquivos. $\mathrm{O}$ mesmo jornalista tornou-se, então, um dos principais tradutores de Djambul.

6 - Você acredita que os tradutores da era soviética podiam, de alguma forma, influenciar a publicação de determinados escritores, agindo como gatekeepers? Eles podiam pular algumas das regras ao alterar ou omitir trechos, eles tinham algum poder nos encontros da União dos Escritores? Você acha que isto poderia ter mudado no período do "degelo", eles poderiam ter ganhado mais autonomia então?

Os tradutores podiam propor trabalhos para tradução, tentando promover determinados escritores etc. Mas as decisões, claro, eram tomadas em um nível superior, envolvendo as corporações formais da censura. Como mostrado por Samantha Sherry em seu livro supracitado, a censura não era apenas formal, mas onipresente, e envolvia diversas camadas, indo da autocensura do tradutor ao processo de edição, em que um editor experiente frequentemente executava uma censura prévia que envolvia cortes, reescritas, omissões e práticas similares. O editor, por sua vez, frequentemente tinha que negociar certas escolhas com outras pessoas, e assim por diante, até que o manuscrito pudesse ser aprovado, novamente por um órgão formal de censura. Então seria mais exato dizer que a função do gatekeeper era realizada por este processo de diversas camadas de censura. 
7 - No Brasil, quase até o final do século passado, a maioria das traducões de literatura russa era feita a partir de traducōes com intermediários do francês ou inglês. Algo similar ocorreu em países nórdicos ou escandinavos? Você também pesquisou a prática soviética em traduções com intermediários. Quais foram suas principais conclusões?

Sim, nos períodos iniciais (final do século 19 e início do século 20) muitas obras literárias russas chegaram aos países nórdicos por meio de traduções alemãs. Esta tradução indireta do russo foi praticamente abandonada na primeira década do século 20. Mas práticas indiretas continuaram a ser a norma quando o assunto é a literatura de línguas das repúblicas soviéticas. Esses trabalhos são, em geral, traduzidos de uma tradução russa. Como citado anteriormente, estudei o uso de interlineares de língua russa em tradução das línguas de nacionalidades para o russo e entre essas próprias línguas. Minha conclusão é a de que, ainda que a prática tenha sido considerada inferior e medidas administrativas tenham sido tomadas repetidamente contra isso, ela persistiu até o final da era soviética como parte indispensável do funcionamento do sistema da literatura soviética.

8 - Ao escrever sobre as pseudotraduções na URSS, você tange o vasto uso de elementos estrangeirizantes derivados de linguas das ex-repúblicas soviéticas como um modo usado pelo pseudotradutor para marcar o fato de que o texto era realmente originário de literatura não russa (e que existia mesmo). Mas os mesmos elementos estrangeirizantes são empregados também nas traducões para o russo das obras do brasileiro Jorge Amado por seu tradutor soviético, Iúri Kalúguin. Neste caso, entretanto, os regionalismos brasileiros soam estrangeiros até para brasileiros de regiões diferentes da Babia de Amado e do Nordeste do Brasil. Como você explicaria o uso deste tipo de elementos estrangeirizantes em literatura soviética "progressista" dos anos 1950 e 1960?

É uma observação muito interessante! Talvez isto pudesse estar ligado ao estudo de Brian Baer sobre o acondicionamento das traduções "progressistas" do Terceiro Mundo no periódico Inostrânnaia literatura nos anos 1950, que era frequentemente nacionalista, salientando a peculiaridade cultural dessas nações (ver Baer, Brian, "From International to Foreign: Packaging Translated Literature in Soviet Russia," Slavic and East European Journal, 60.1, 2016).

9 - Vocêpoderia definir brevemente a literatura estrangeira traduそ̨ida para o russo e a traduzida para as línguas das repúblicas russas e as zonas de influência russas durante a era soviética? O que diferencia esses fluxos nas politicas culturais do Partido, em sua opinião? 
Os clássicos da literatura mundial (inclusive literatura infantil) eram, claro, traduzidos e retraduzidos durante todo o período. A literatura moderna "progressista" constituiu um cânone tradutório que frequentemente colocava em primeiro plano autores que não eram conhecidos em seus países de origem, como Howard Fast durante o início dos anos 1950 (o mais traduzido de todos os autores estrangeiros da URSS!). Durante os anos 1960, autores modernos ocidentais que não eram tão progressistas e até modernistas (Salinger, Auden, Kafka) podiam chegar até os leitores russos. Katerina Clark escreve de maneira interessante sobre os esforços soviéticos nos anos 1930 para maquinar uma literatura mundial centrada em Moscou por meio de traduções em russo e do russo (Moscow, The Fourth Rome: Stalinism, Cosmopolitanism, and the Ecolution of Soviet Culture, 1931-1941, 2011). Quanto às repúblicas, um ponto importante é que muita literatura estrangeira foi traduzida em suas diversas línguas de modo indireto por meio de traduções russas das obras em questão. A tradução russa (já censurada), deste modo, funcionava como um gatekeeper.

10 - A literatura de Jorge Amado também traz inúmeras menções a rituais religiosos (cristãos, negros etc.) e Amado era conbecido praticante de religiões africanas, bastante difundidas em seu Estado natal, a Babia. Essas menç̃oes não são suprimidas nas traduções russas, por exemplo, na primeira tradução de "Gabriela, cravo e canela", de 1961, ao contrário do que se poderia esperar, enquanto alguns trechos mais sensuais de sua obra são omitidos (ao mesmo tempo em que outros, na mesma obra, são mantidos e outros ainda, intensificados) e mençoes a Lênin ou ao tsar são completamente cortadas. Você poderia citar os casos mais interessantes, a seu ver, envolvendo cortes e omissões ou a manutenção inesperada de elementos que poderiam ser contrários à ideologia do Partido (considerando ainda a anedota de que "na URSS não havia sexo" e a situação em que ela nasceu)?

Um caso interessante é o da tradução de 1960 de Rita-Rait Kovaliôva do romance de J. D. Salinger O Apanhador no Campo de Centeio, que representou muitas dificuldades à tradutora soviética em termos de gírias, palavrões, franqueza sexual etc. A tradutora desenvolveu suas próprias e eficientes estratégias de transmitir tais elementos sem cortes. Como mostrado por Aleksêi Semenenko, "ao invés de escolher a solução fácil e frequentemente usada de eliminar e/ou substituir as obscenidades com eufemismos, na maioria dos casos Rait cria expressões ad hoc que produzem um efeito de desfamiliarização, fazendo o leitor parar e adivinhar o significado do contexto" (SEMENENKO, Aleksei, "Smuggling the other: Rita Rait-Kovaleva's Translation of J. D. Salinger's The Catcher in the Rye," Translation 
and Interpreting Studies, Special Issue: Contexts of Russian Literary Translation, ed. By Julie Hansen and Susanna Witt, 11.1, 2016).

11 - Em seus trabalhos, você usa muitas informações e citações dos arquivos de Moscou, principalmente do RGALI. Não existem tantos artigos e livros sobre casos e historiografia dos Estudos da Traducão russos em inglês, espanhol, português, pelo menos de acordo com minhas pesquisas, como sobre os Estudos da Tradução acerca de outras línguas, que não a russa, apesar de haver trabalhos importantes sobre os Estudos da Traducão na Rússia em língua russa, como Literalistas Derrotados, de Azóv etc., além de dezenas de artigos sobre casos, autores on textos especificos. Apesar disto, Ažón, por exemplo, já deixou suas atividades acadêmicas. Você acha que a academia russa deveria se esforçar mais para lidar com o problema da falta de trabalhos nesta área ou haveria alguma falta de interesse nela, talvez.por motivos politicos? Você concorda que há uma deficiência em sistematizar esses estudos porparte dos pesquisadores russos? Acha que ainda há muito terreno virgem para ser explorado por pesquisadores internacionais nesta área?

Sim, absolutamente, ainda há muito por fazer por parte dos pesquisadores, tanto internacionais como russos, nesta área. Há muito material nos arquivos relativo ao trabalho e à vida de tradutores individuais, assim como a instituições, por exemplo, as editoras. Ainda que tenha surgido um estudo alemão da editora Vsemírnaia literatura ${ }^{25}$, o grande empreendimento tradutório da década de 1920 e a editora Academia, um dos principais estabelecimentos para literatura traduzida no final dos anos 1920 e início dos 1930, tenho certeza de que há muito relacionado a elas (o estudo em questão é de Tatiana Bedson e Maxim Schulz, Sonjetische Übersetzungskultur in den 1920er und 1930er Jahren: Die Verlage V semirnaja literatura und Academia, 2015). Sei que há pesquisas muito interessantes sendo realizadas na Universidade Estatal de Moscou e na Escola Superior de Economia (Moscou), assim como em São Petersburgo (por exemplo, na Casa Púchkin), mas seria certamente uma boa ideia fazer um balanço das pesquisas realizadas em outros lugares para se ter uma ideia melhor. Não acho que haja uma falta de interesse por motivos políticos.

12 - Você concorda que, até certo ponto, os arquivos russos, apesar de seu passado obscuro poderiam hoje em dia ser considerados muito mais abertos que em muitos outros paises (desenvolvidos) do mundo com o volume de documentos soviéticos já abertos a um amplo público, inclusive estrangeiro? 
Não estou certa sobre o ponto dos arquivos russos serem muito mais abertos que outros. É verdade que muitos arquivos russos estão abertos a pesquisadores, inclusive estrangeiros, mas outros são menos acessíveis hoje que há 20 anos - por exemplo, os arquivos da KGB, que seriam de grande interesse para pesquisa sobre muitos pesquisadores individualmente. E ainda há muita burocracia e dificuldades em se obter cópias de material de arquivo.

13 - Qual o status dos Estudos da Tradução russos em países nórdicos atualmente?

$\mathrm{Na}$ Suécia, há alguns pesquisadores acadêmicos que conduzem pelo menos alguma pesquisa dentro dos Estudos da Tradução Russos. Na última década, tivemos algumas teses de doutorado: Hamlet, o Sinal: Traduções Russas de Hamlet e a Formação do Cânone Literário ${ }^{26}$ [tradução nossa] (Estocolmo, 2007), de Aleksei Semenenko, e Os sonetos de Shakespeare em Russo: o Desafio da Tradução ${ }^{27}$ [tradução nossa] (Umeå, 2017), de Elena Rassokhina, e também uma dedicada a traduções do russo para o sueco, Janela para o Leste: a Literatura russa em tradução para o sueco com um estudo de caso da recepção sueca de Nikolai Gógol ${ }^{28}$ [tradução nossa] (em sueco, Uppsala, 2012), de Nils Håkanson. Mas isto é mais parte dos Estudos da Tradução suecos... Quanto a outros países nórdicos, a Universidade da Finlândia Oriental em Joensuu é, talvez, o único lugar onde o setor está bem estabelecido.

14 - Você esteve entre os organizadores da conferência Tradução em Contextos Russos ${ }^{29}$ [tradução nossa], em Uppsala, Suécia, e a série de mesas-redondas e conferências dedicadas aos Estudos da Tradução Russos e Eslavos nas conferências da ASEEES em 2015 e 2016. Como foi organizar tais eventos? E quais outros projetos você tem ligados a esta área de pesquisa para o futuro? Algum outro livro a ser lançado em breve também?

Tenho organizado diversos painéis em assuntos relacionados na ASEEES desde 2011, mas o formato de "série" foi introduzido em 2016 e continuado neste ano, em Chicago (2017). Acredito que este seja um modo muito frutífero de continuar a colaboração dentro da rede que mencionei acima. Temos tentado

26 Hamlet the Sign: Russian Translations of Hamlet and Literary Canon Formation.

27 Shakespeare's Sonnets in Russian: the Challenge of Translation.

28 Window to the East: Russian literature in Swedish translation with a case study of the Swedish reception of Nikolai Gogol.

29 Translation in Russian Contexts. 
isso em outras conferências também, como a ICCEES, o Congresso Nórdico de Eslavistas e a Primeira e Segunda Conferência Anual de Tartu sobre Estudos Russos e do Leste Europeu (em 2016 e 2017). Meu projeto de pesquisa atual, “The Interface with the Foreign: The 'Soviet School of Translation,' Cold War and World Literature, 1945-1985" é financiado pelo Conselho de Pesquisa Sueco para o período de 2015-2018 e, espera-se, deve resultar em uma monografia.

15 - Atualmente como você caracterizaria as traducõoes literárias para o russo? Elas seguem alguma linha especifica, em sua opinião?

Infelizmente, não tenho tempo para monitorar a área de traduções contemporâneas para o russo. Mas seria interessante analisar a situação atual tendo em vista o pano de fundo da anteriormente hegemônica "escola soviética de tradução" com seu ímpeto bastante domesticador.

\section{Interview with Susanna Witt: Soviet translation history was a sensitive subject and therefore neglected}

Marina Darmaros

Susanna Witt is graduated in Slavic Studies and defended her $\mathrm{PhD}$ thesis on the creation of Doctor Zhivago by Pasternak, in 2001, at the University of Stockholm, where she is currently an associate professor and lecturer. Since then, she is devoted to Translation Studies, focusing on the Soviet period.

1 - Can you tell us about your background and how did you start in Russian Translation Studies? Was it a though way to go through in Translation Studies specifically in the Russian/ Soviet reality? 
My background is in Slavic Studies with a specialization in Russian literature. I defended my PhD, Creating Creation: Readings of Pasternak's Doktor Zhivago, at Stockholm University in 2001 and began publishing in the field of Russian literary modernism. Boris Pasternak was also a renowned translator and later I wrote an article on his 1940 rendition of Shakespeare's Hamlet. As a result, I got interested in the broader significance of literary translation as a cultural phenomenon in Russia, and the Soviet Union in particular, but was surprised to find out that the topic was largely neglected in (western) Slavic Studies and virtually absent from the central publications of modern Translation Studies. This was around the mid-2000s. In Russia itself, there has of course been a long and strong tradition of studying and theorizing translation, but it has been monolingually Russophone, and during Soviet times it often had a normative and heavily ideologized approach. For ideological reasons the history of translation of that period was a sensitive topic and thus neglected. Late Soviet and post-Soviet translation scholarship has largely been linguistically based and prescriptive in character. These blind spots are the reason why I decided to address the issue in a project, "Totalitarianism and Translation: Control and Conflict in Soviet Translation Practices, 1932-1953," aimed to establish literary translation as an object of research within studies of the culture of the Stalin period. During the years 2009-2013 I worked extensively in Russian archives, which was a new experience for me and a really tough one, for sure. In some sense I felt that not so much had changed since Soviet times (during which I spent a year at Moscow University), despite the rapid and obvious modernization of the cityscape and everyday life.

2 - In your paper "The art of accommodation: The First All-Union Conference of Translators", from 2013, you write: "One aspect of Soviet culture still largely neglected, however, is that of literary translation". Do you believe these studies are still neglected or are they rising?

I would say that by now the picture has changed radically (especially bearing in mind that the article you mention was written some years prior to its publication in 2013). Within the overall broadening of geographical perspectives that translation studies have seen over the last decades (think about the large body of scholarship on translation relating to India, China, Japan, Africa, the Americas) and which has been challenging the traditionally Eurocentric priorities of the discipline, Russia and Eastern Europe have at last gained visibility. This is due to international collaboration, conferences and publication initiatives which have gathered scholars across disciplines and national borders. In 2008 I attended the first major conference in the field, organized by Emily Lygo at Exeter university, Great Britain ("Pushkin's Post Horses: Literary Translation in Russian Culture"). 
It brought together scholars from Russia, Europe and America, several of whom were to be instrumental in promoting research in the field, above all Brian James Baer who published his edited volume Contexts, Subtexts and Pretexts: Literary Translation in Eastern Europe and Russia in 2011. To this book I also contributed a chapter, presenting the first results of my project. The Exeter conference itself resulted in the 2013 volume The Art of Accommodation: Literary Translation in Russia (eds. Leon Burnett and Emily Lygo). In 2014 I organized an international conference ("Translation in Russian Contexts: Transcultural, Transliteral and Transdisciplinary Points of Departure") at Uppsala University, Sweden, together with my colleague Julie Hansen. Its 60 participants from 15 countries subsequently made up the core of an expanding network of scholars in the field which by now includes over a hundred researchers. A volume based on selected contributions to the Uppsala conference has just appeard (Brian James Baer, and Susanna Witt (eds.), Translation in Russian Contexts: Culture, Politics, Identity, London: Routledge, 2018). I would like to mention as well Andrea Ceccherelli, Lorenzo Costantino, Cristiano Diddi (eds.) Translation Theories in the Slavic Countries, Salerno 2015. It is a special issue of the journal Europa Orientalis, vol. XXXIII (2014), based on a conference held almost simultaneously with our Uppsala conference in 2014. As a sign of a transdisciplinary breakthrough, Russian-themed special issues of translation journals such as Translation and Interpreting Studies (11:1, 2016) and Translation Studies (11:2, 2018) are appearing while, for example, Slavic and East European Journal recently featured a "forum" on translation in Russia $(60: 1,2016)$. There has also been an increase in interest towards Russian scholarship on translation, as witnessed by the conference "Going East: Discovering New and Alternative Traditions in Translation Studies" held at Vienna University in 2014 (and the 2016 volume by the same title, edited by Laris Schippel and Cornelia Zwischenberger). Noticable here is also Birgit Menzel's and Irina Alekseeva's edited volume Russische Übersetzungswissenschaft an der Schwelle zum 21. Jabrhundert which appeared in 2013. So, the body of research on translation and translation scholarship in the Russian context is growing steadily (including an increasing number of $\mathrm{PhD}$ theses), although there are still obstacles such as the relatively closed systems of circulation: French, German and Englishlanguage scholarship still tend to ignore each other while Russia-based studies often operates without references to international scholarship.

3 - What are the main researchers in this area nowadays in your opinion? And which texts you consider canonical and would recommend to anyone that has some basis by and large in Translation Studies and is wishing to make his way towards Russian Translation Studies? What advice would you give to anyone starting to path his way in Russian Translation Studies? 
Above I have already mentioned some names. When it comes to literary translation in the Russian context, one of the central figures is, undoubtedly, the above-mentioned Brian James Baer, who published his monograph Translation and the Making of Modern Russian Literature in 2016. As for the larger picture of the history of translation in Russia I would like to mention Sergey Tyulenev's book Translation and the Westernization of Eighteenth Century Russia: A Social-Systemic Approach (2012). As for archival-based research (made possible with the break-up of the Soviet system), one should mention Andrei Azov's 2013 monograph Poverzhennye bukvalisty: Iz istorii khudozhestvennogo perevoda v SSSR v 1920-1960-e gody [The defeated literalists. From the history of literary translation in the USSR 1920s through the 1960s]. My own research, partly based on the same source material, has many points in common with his, although my approach is broader and includes translation analysis proper. Interesting research is also being conducted on the Soviet journals that presented foreign literature in Russian translation, the periodicals Internatsional'naia literatura [International literature] (1933-1943) and Inostrannaia literatura [Foreign literature] (1955-). The former is the issue of a research project at Higher School of Economics in Moscow conducted by my network colleagues Elena Zemskova and Elena Ostrovskaia and focusing on institutional and personal aspects of the journal's activities. This journal has also been studied by Nailya Safiullina and Rachel Polonsky, who focused, inter alia, on the reception of these texts in Stalin's Russia. A valuable contribution here is also Samantha Sherry's 2015 monograph Discourses of Regulation and Resistance: Censoring Translation in the Stalin and Khrushchev Era Soviet Union, a comparative study of the two journals. As for "canonical texts" Maurice Friedberg's pioneering (although not archival-based) studies should be mentioned here: A Decade of Euphoria. Western Literature in Post-Stalin Russia, 1954-64 (1977) and Literary Translation in Russia. A Cultural History (1997). Soviet theory of translation is selectively presented in Lauren Leighton's likewise pioneering 1991 book Two Worlds, One Art: Literary Translation in Russia and America. Some classics of Soviet-Russian translation scholarship are accessible in other languages: Kornei Chukovskii's classical Vysokoe iskusstvo has been translated by Lauren Leighton as The Art of Translation: Kornei Chukovskii's "The High Art" (1984), Efim Etkind, one of the most astute Russian translation scholars, is represented in French by the volume Un art en crise: essai de poétique de la traduction poétique (1982, trans. Wladimir Troubetzkoy). Brian James Baer is now translating Andrei Fedorov's seminal Foundations for a general theory of translation (first ed. 1953) and has previously published Russian Writers on Translation: An Anthology (2013, together with Natalia Olshanskaia). At present, I am myself together with Baer preparing a "Soviet history of translation in documents," making accessible texts from the early 1920 s and on. 
My advice to anyone "starting to path his way in Russian Translation Studies" would perhaps be to stay aware of the fact that translation in Russia always has been "more than translation" (to paraphrase the traditional saying that in Russia, "a poet is more than a poet"): since translation is the physical manifestation of an encounter with "the foreign" it has always been a sensitive activity in a society which has more often than not been informed by suspicion and has guarded itself through history with travel regulations and the like. A site of negotiation, contestation and resistance, Russian translation has always to be contextualized in order to be conceptualized. Which is of course true for translation in general, but perhaps even more important here.

4 - In many of your works you study cases that involve languages in which you are not native, as I understand (as in Dzhambul or Pasternak translating from Lachian). How do you choose the cases you are going to research, presuming that the language barrier is bigger in this sense?

True. There are obvious limitations as to the number of languages a researcher may reasonably know. My own native language is Swedish, I have fluency in Russian and English, plus reading ability in French, Polish and Ukrainian. But I would say that all depends on what type of research you want to do. The cases you mention are very different, although united by the fact that both are mediated and not direct translations. They are both produced with the help of a Russian-language interlinear, a so-called podstrochnik (literally meaning 'under the line'), a very common Soviet practice, to which I have devoted several studies. So the Soviet translators themselves did not always know (in fact, very often they didn't) the languages of the original, especially when it came to translations from the many languages of the Union itself. As for Dzhambul, the original Kazakh texts of whom are largely lacking or disputable, my main focus was the function and significance of these translations in their context. I was also able to pinpoint the institutional handling of them and to sketch the probable production process, which appeared to be a collective enterprise resulting in translations as well as original Kazach texts. In the case of Pasternak's translation of the Lachian poet Óndra Lysohorsky, I found the original texts in Pasternak's archive, as well as Łysohorsky's own Russian-language interlinear. I was able to read the original thanks to my knowledge of Polish since Lachian, a dialect that Lysohorsky himself promoted as a language, is something in between Polish, Czech and Slovak. I could assess the closeness of the interlinear to the original and then analyze Pasternak's translation from it. Detailed translation analyses I have carried out 
only from languages I know, such as Georgii Shengeli's rendition of Byron's Don Juan (Witt, Susanna, "Translation and Intertextuality in the Soviet-Russian Context: The Case of Georgii Shengeli's Don Juan." Cluster on Russian translation, ed. by Brian James Baer. Slavic and East European Journal, 60:1, 2016).

5 - Could you punctuate some of the particular characteristics of Russian Translation Studies that differ from these studies on other languages/realities? "Literalism", "free translation", "realist translation", "naturalism", "realist translation" are, in my view, features yet to be discovered by many researchers not concentrated in the Russian field of these studies, do you agree? Could you punctuate as well some of the most interesting cases on Russian Translation Studies in your opinion? In your researches there are mentions to the Maimbet pseudotranslation case, or the brief discussion started by Gideon Toury on the Drhambul case as an example of sculture planning>... Could you speak a little about it?

You're right, of course these notions are not exclusively Russian (except perhaps for the "realist translation," which to my knowledge have not been conceptualized elsewhere). But a distinguishing feature in the Soviet context was the ideologization of norm, tying certain approaches to translation to ideologically reprehensible positions and defining the limits of translation discourse. It must be remembered that here, at certain periods, such issues were not purely academic, but could send people to the camps.

GideonToury (2005) was the first to bring Dzhambul, the Kazakh bard of Stalinism, into western translation scholarship. But his only source for theorizing what he called pseudotranslation in this case were the memoirs of composer Dmitrii Shostakovich, a text which in itself is somewhat debated. Inoficially, in the Soviet context, the Dzhambul case was also habitually referred to as fakelore, a thing taken for granted. I decided that the case deserved more thorough treatment as indicative of Soviet translation practices and attitudes to translation in an imperial context. In the process I discovered quite a few similar cases, in which "nationalities" poets published mainly in Russian translation were promoted as representatives of their native literatures to be included in the Soviet canon. This is what I, using Toury's and Even Zohar's concept of "culture planning" refer to as "culture planning from below" since the (Russian) translators themselves were often instrumental in publishing these texts. The case of Maimbet is an extreme one because not only were there no original texts, there was no original poet either. This Kazakh figure was launched in 1935 by a local Russian journalist in Alma-Ata. His translations of Maimbet appeared even in the central newspaper Pravda, supplied with a photo of the bard, a typical Central Asian man with his 
traditional string instrument. At the time there was great demand (created from above) for "nationalities" poets to represent their republics at cultural festivals in the larger Russian cities. So the journalist was approached by the authorities and asked to bring his poet to Moscow for the Kazakh festival in 1936. His solution to this problem was to announce that Maimbet, as a member of a nomadizing family, had crossed the border to China and remained there. The imaginary status of Maimbet was confirmed in a letter of denunciation from the journalist's colleagues at the local writers' union which I found in the archive. The same journalist then became one of the principal translators of Dzhambul.

6 - Do you believe that translators from the Soviet era could somehow influence the publication of some specific writers, acting as gatekeepers? Could they skip some of the rules by changing or omitting excerpts, did they have any power in the Writers Union's meetings? Do you believe this could have changed in the period of the "Thaw", could they have gained more autonomy then?

Translators could propose works for translation, trying to promote certain writers etc. But the decisions were of course taken at higher levels, involving the formal bodies of censorship. As shown by Samantha Sherry in her abovementioned book, censorship was not only formal but ubiquitous and multilayered, stretching from the selfcensoring of the translator to the editing process in which the experienced editor often carried out preemptive censorship involving excisions, rewritings, omissions and the like. The editor, in turn, often had to negotiate certain choices with other people, and so on, until the manuscript could be approved, again by the formal body of censorship. So it would perhaps be more precise to say that the function of gatekeeper was carried out by this multilayered process of censorship.

7 - In Brazil, almost until the end of the last century, most of the translations from Russian literature were done through retranslations from French and English. Was there anything alike in Nordic or Scandinavian countries? You researched as well the Sovietpractice in retranslations. What were your main conclusions on it?

Yes, in the early periods (late 19th and early 20th century) many works of Russian literature reached the Nordic countries via German translations. Such indirect translation from Russian was practically abandoned in the first decade of the 20th century. But indirect practices continued to be the norm when it came to literature from the languages of the Soviet republics. Such works were generally 
translated from a Russian translation. As mentioned above, I have studied the use of Russian-language interlinears in translation from nationalities languages into Russian and between such languages themselves. My conclusion was that even if the practice was deemed inferior from the very beginning and administrative measures were repeatedly taken against it, it persisted until the end of the Soviet era as an indispensable part of the functioning of system of Soviet literature.

8 - When writing about pseudotranslations in USSR, you touch the vast use of foreignizing elements derived from languages from former Soviet republics as a way used by the psendotranslator to mark that the text is in fact originated from a non-Russian literature (and that it indeed exists). But these same foreignizing elements are employed as well in the translations into Russian of Brazilian Jorge Amado's works by his Soviet translator, Yuri Kalugin. In this case, however, the Brazilian regionalisms sound foreign even to Brazilians from areas other than Amado's Babia and Brazilian northeast. How would you explain the use of such kinds of foreignizing elements in "progressive" Soviet literature of the 1950s and 1960s?

This is a very interesting observation! Perhaps it could be related to Brian Baer's study of the packaging of "progressive" translations from the Third World in the journal Inostrannaia literatura during the 1950s, which was often nationalist, underscoring the cultural peculiarity of these nations (see Baer, Brian, "From International to Foreign: Packaging Translated Literature in Soviet Russia," Slavic and East European Journal, 60.1, 2016).

9 - Could you define briefly the foreign literature translated into Russian and the one translated into the Russian republics languages and Russian influence zones during the Soviet era? How do these influxes differ in the cultural policies of the Party as you see it?

The classics of world literature (including children's literature) were of course translated and retranslated during the whole period. "Progressive" modern literature made up a translational canon which often foregrounded authors who were not well known in their native countries, such as Howard Fast during the early 1950s (the most translated of all foreign authors in the USSR!). During the 1960s modern western authors who were not so progressive and even modernist (Salinger, Auden, Kafka) could find their way to Russian readers. Katerina Clark writes interestingly about the Soviet efforts in the 1930s to devise a Moscow-centered world literature by ways of translations into and from Russian (Moscow, The Fourth Rome: Stalinism, Cosmopolitanism, and the Evolution of Soviet Culture, 1931-1941, 
2011). As for the republics, an important thing is that much foreign literature was translated into their various languages in an indirect way via the Russian translations of the works in question. The Russian (already censored) translation thus functioned as a gatekeeper.

10 - Jorge Amado's literature also brings countless mentions to religious rituals (Christian, black etc.) and Amado was a well-known practitioner of African religions that are widespread in his home state, Babia. These mentions are not suppressed in the Russian translations, for example, in the first translation of "Gabriela, clove and cinnamon", of 1961, on the contrary of what one could expect, while some, more sensual excerpts of his works are omitted (at the same time as others, in the same works, are kept, and even others are intensified) and mentions to Lenin or the tsar are completely cut. Could you mention the most interesting cases, in your view, involving cuts and omissions or the unexpected maintenance of elements that could be contrary to the Party's ideology (considering further the anecdote that "in USSR there wasn't sex" and the situation that gave birth to it)?

An interesting case is Rita Rait-Kovaleva's 1960 translation of J.D. Salinger's novel The Catcher in the Rye, which presented many difficulties to the Soviet translator in terms of slang expressions, foul language, sexual explicitness, etc. The translator developed her own effective strategies in conveying such things without excisions. As shown by Aleksei Semenenko, "instead of choosing the often-used easy solution of eliminating and/or replacing the obscenities with euphemisms, in most instances Rait creates ad hoc idioms that produce an effect of defamiliarization, making the reader pause and guess the meaning form the context." (Semenenko, Aleksei, "Smuggling the other: Rita Rait-Kovaleva's Translation of J.D. Salinger's The Catcher in the Rye," Translation and Interpreting Studies, Special Issue: Contexts of Russian Literary Translation, ed. By Julie Hansen and Susanna Witt, 11.1, 2016)

11 - In your works, you use many information and quotes from the Moscow archives (above all RGALI). Russian Translation Studies in English, Spanish, Portuguese (at least according to my researches) aren't as much abundant of papers and books dedicated to its cases and historiography as Translation Studies on languages other than Russian, though there are important works on Russian Translation Studies in Russian, such as Azov's "Poverzhenie bukvalisti" etc., besides dozens of articles on specific cases, authors or texts. Even though, the young Azov, for example, has already left his scholar activities. Do you believe Russian academy has to make bigger efforts to tackle the lack of works in this field or would there be a lack of interest on it, maybe for political reasons? Do you agree there's a deficiency in systematizing these studies by 
Russian researchers? Do you believe there are still a lot of virgin territories to be explored by international researchers in this area?

Yes, absolutely, there is still so much to do for researchers, international as well as Russian, in this area. There is a lot of material in the archives pertaining to the work and life of individual translators as well as to institutions such as publishing houses. Even if there has appeared a German study of Vsemirnaia literatura [World literature] publishing house, the great translation enterprise of the 1920s, and the publishing house Academia, a main outlet for translated literature in the later 1920s and early 1930s, I am sure that there is a lot more to do with them. (The study i question is Tatiana Bedson and Maxim Schulz, Sowjetische Übersetzungskultur in den 1920er und 1930er Jahren: Die Verlage Vsemirnaja literatura und Academia, 2015). I know that interesting research is going on at Moscow State University and Higher School of Economics (Moscow), as well as in St. Petersburg (for example, at the Pushkin House), but it would surely be a good idea to take stock of research carried out elsewhere to get a better picture. I don't think there is a lack of interest for political reasons.

12 - Do you agree that, at some extent, that the Russian archives, despite its obscure past, could be nowadays considered much more open than in many other (developed) countries of the world with the volume of Soviet files that are already open for a wide public, including foreign?

I'm not sure about the point of Russian archives being much more open than other ones. It is true that many Russian archives are open to researchers, including foreigners, but other ones are less accessible now than 20 years ago for example, the KGB archives, which would be of great interest for research on many individual translators. And still there is a lot of bureaucracy and difficulties in obtaining copies of archival material.

\section{3 - What is the status of Russian Translation Studies in Nordic countries currently?}

In Sweden there is a handful of scholars who conduct at least some research within Russian Translation Studies. For the last decade we have had a couple of PhD theses: Aleksei Semenenko's Hamlet the Sign: Russian Translations of Hamlet and Literary Canon Formation (Stockholm, 2007) and Elena Rassokhina's Shakespeare's Sonnets in Russian: the Challenge of Translation (Umeå, 2017) and also one devoted 
to translations from Russian into Swedish, Nils Håkanson's Window to the East: Russian literature in Swedish translation with a case study of the Swedish reception of Nikolai Gogol (in Swedish, Uppsala, 2012). But that is rather part of Swedish Translation Studies... As for the other Nordic countries, the university of Eastern Finland at Joensuu is perhaps the only place where the field is well established.

14 - You were among the organizers of the conference "Translation in Russian Contexts," in Uppsala, Sweden, and the series of round-tables and conferences dedicated to Russian and Slavic Translation Studies in the ASEEES conferences in 2015 and 2016. How was it to organize such events? And which other projects do you have connected to this field of research for the future? Any other books coming up soon?

I have been organizing various panels on related topics at the ASEEES since 2011, but the "series" format was introduced in 2016 and continued this year in Chicago (2017). I think this is a very fruitful way of continuing collaboration within the network mentioned above. We have tried it at other conferences as well, such as the ICCEES, the Nordic Congress of Slavists and the First and Second Annual Tartu Conference on Russian and East European Studies (in 2016 and 2017). My current research project, "The Interface with the Foreign: The 'Soviet School of Translation,' Cold War and World Literature, 1945-1985” is funded by the Swedish Research Council for the period 2015-2018 and will hopefully result in a monograph.

15 - Currently, how would you characterize the literary translations into Russian? Do they follow an specific line on your opinion?

Unfortunately, I don't have time to monitor the field of contemporary translations into Russian. But it would be interesting to analyze the current situation against the background of the previously hegemonic "Soviet school of translation" with its generally quite domesticating thrust. 